\title{
Does self-perceived oral health status have an impact on nutrient intake amongst adults at a high risk of cardiovascular disease in Northern Ireland?
}

\author{
S.M. Wallace, R. O’Neill, L. McGowan, C. McEvoy, G. McKenna, M. McKinley, M. Cupples, \\ C. Patterson, I. Young, F. Kee and J.V. Woodside \\ Centre of Excellence for Public Health, Queen's University Belfast, United Kingdom, BT12 6BA
}

Data from the randomised controlled trial PREDIMED observed a significant reduction in the incidence of T2DM and CVD, the leading causes of death worldwide, in response to adoption of a Mediterranean style diet (MD) ${ }^{(1,2)}$. Oral health status has been shown to impact dietary choice and nutritional status; older adults who wear dentures or have fewer natural teeth present tend to consume poor quality diets which are low in fibre and protein, and high in complex carbohydrates and calories ${ }^{(3)}$.The aim of this preliminary analysis is to analyse baseline data from a 12-month pilot cluster randomised study evaluating a peer support MD intervention in Northern Ireland (NI), to investigate whether self-perceived oral health status has an impact on nutrient intake.

Participants in NI were eligible to participate if they were within a community group, were aged $\geq 40$ years, had a Mediterranean Diet Score $\leq 5$ and had at least one cardiovascular disease risk factor, which included having Type 2 Diabetes Mellitus and not being on any medication, being a current smoker and having hypertension (high BP) systolic BP $>=140$ or diastolic $>=90 \mathrm{mmHg}$. Dietary data from 4-day food diaries collected from these participants $(n=31$, female $n=22(71 \%)$, mean age 59.5y) were entered into Nutritics and analysed using SPSS v22. Mean intake of energy and macro- and micronutrients were calculated, and ANOVAs were conducted to investigate whether mean daily intake of the nutrients differed between individuals with different self-perceived oral health status, assessed via questionnaire, at baseline.

Significant differences in mean intake of total fat (g/day), energy (kcal/day) and MUFA (g/day) were observed ( $\mathrm{p}$ values from ANOVA $p=0.04, p=0.03$ and $p=0.03$ respectively) between the three self-perceived oral health groups (excellent or very good $n=12$, good $n=8$, fair or poor $n=11$ ); with post-hoc analysis demonstrating that the intake of total fat, energy and MUFA of those with fair or poor oral health were significantly higher than those with good oral health ( $\mathrm{p}$ values from Bonferroni analysis $\mathrm{p}=0.03, \mathrm{p}=0.04, \mathrm{p}=0.02$ respectively). No significant differences were observed between those individuals with excellent or very good oral health and those with either good or fair or poor oral health ( $\mathrm{p}$ values from Bonferroni analysis $\mathrm{p}>0 \cdot 05$ ).

\begin{tabular}{|c|c|c|c|c|c|c|c|}
\hline \multirow[b]{3}{*}{ Mean Daily Energy and Nutrient Intakes } & \multicolumn{6}{|c|}{ Self-reported Oral Health Status } & \multirow[b]{3}{*}{$\mathrm{p}$ value } \\
\hline & \multicolumn{2}{|c|}{$\begin{array}{l}\text { Excellent or Very Good } \\
(\mathrm{n}=12)\end{array}$} & \multicolumn{2}{|c|}{$\begin{array}{c}\text { Good } \\
(\mathrm{n}=8)\end{array}$} & \multicolumn{2}{|c|}{$\begin{array}{l}\text { Fair or Poor } \\
\quad(\mathrm{n}=11)\end{array}$} & \\
\hline & Mean & $\mathrm{SD}$ & Mean & SD & Mean & $\mathrm{SD}$ & \\
\hline Energy (kcal) & $1731 \cdot 67$ & $578 \cdot 65$ & $1504 \cdot 36^{\mathrm{a}}$ & $443 \cdot 70$ & $2181 \cdot 34$ & $562 \cdot 86$ & 0.03 \\
\hline Carbohydrate (g) & $195 \cdot 61$ & $66 \cdot 46$ & $182 \cdot 05$ & $46 \cdot 51$ & $229 \cdot 74$ & $60 \cdot 46$ & $0 \cdot 21$ \\
\hline Fat $(\mathrm{g})$ & $73 \cdot 26$ & $31 \cdot 36$ & $54 \cdot 07^{\mathrm{a}}$ & $24 \cdot 01$ & $90 \cdot 03$ & 27.95 & 0.04 \\
\hline Protein $(g)$ & $67 \cdot 04$ & $15 \cdot 51$ & $62 \cdot 32$ & $16 \cdot 78$ & $81 \cdot 29$ & 21.41 & 0.07 \\
\hline MUFA (g) & $25 \cdot 05$ & $9 \cdot 62$ & $18 \cdot 50^{\mathrm{a}}$ & $8 \cdot 59$ & $31 \cdot 57$ & $10 \cdot 59$ & 0.03 \\
\hline EPA $(g)$ & 1.42 & 1.84 & 3.65 & 3.82 & $4 \cdot 18$ & $3 \cdot 57$ & 0.40 \\
\hline DHA (g) & $1 \cdot 80$ & $2 \cdot 16$ & $3 \cdot 33$ & 3.07 & 2.97 & $2 \cdot 70$ & $0 \cdot 96$ \\
\hline Carotene (ug) & 1842.95 & $1277 \cdot 37$ & $2643 \cdot 24$ & $1812 \cdot 51$ & $2462 \cdot 77$ & $2685 \cdot 23$ & 0.64 \\
\hline Vitamin C (mg) & $55 \cdot 20$ & $39 \cdot 61$ & 86.63 & $44 \cdot 54$ & $66 \cdot 92$ & $51 \cdot 54$ & $0 \cdot 33$ \\
\hline
\end{tabular}

$\mathrm{P}$ value obtained from One-way Analysis of Variance, ${ }^{\mathrm{a}} \mathrm{p}<0.05$ analysed using One-way analysis of variance with Bonferroni correction for comparison of the good oral health status with the fair or poor oral health status groups.

These results highlight differences in nutrient intake according to oral health status. The next stage of this research is to explore oral health status as a predictor of response to the MD intervention. This information can then be used to effectively develop and tailor future dietary interventions, whilst taking account of oral health status, amongst this population group.

1. World Health Organisation, (WHO) (2007).

2. Estruch R et al. (2013) NEJM 368, 1279-90.

3. Moynihan PJ et al. (2007) J. Hum. Nutr. Diet 20, 446-458. 